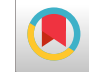

\title{
Post Intensity-Modulated Radiation Therapy Urinary Function for Prostate Cancer; A Prospective Study
}

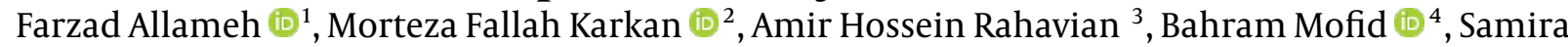
Azghandi $^{4,}{ }^{*}$, Amir reza Abedi ${ }^{5,{ }^{* *}}$, Abbas Basiri $^{1}$ and Saeed Montazeri ${ }^{3}$

${ }^{1}$ Department of Urology, Urology and Nephrology Research Center (UNRC), Shohada-e-Tajrish Hospital, Shahid Beheshti University of Medical Sciences, Tehran, Iran ${ }^{2}$ Department of Urology, Laser Application in Medical Sciences Research Center, Shohada-e-Tajrish Hospital, Shahid Beheshti University of Medical Sciences, Tehran, Iran ${ }^{3}$ Department of Urology, Shohada-e-Tajrish Hospital, Shahid Beheshti University of Medical Sciences, Tehran, Iran

${ }^{4}$ Radiation-Oncology Department, Shohada-e-Tajrish Hospital, Shahid Beheshti University of Medical Sciences, Tehran, Iran

${ }^{5}$ Men's Health and Reproductive Health Research Center, Shahid Beheshti University of Medical Sciences, Tehran, Iran

"Corresponding author: Radiation-Oncology Department, Shohada-e-Tajrish Hospital, Shahid Beheshti University of Medical Sciences, Tehran, Iran. Email: s.azghandi@yahoo.com

${ }^{* *}$ Corresponding author: Men’s Health and Reproductive Health Research Center, Shahid Beheshti University of Medical Sciences, Tehran, Iran. Email: amirezabedi@gmail.com

Received 2020 February 25; Revised 2020 May 05; Accepted 2020 May 30.

\begin{abstract}
Background: At present, there is a lack of evidence concerning urinary complications caused by intensity-modulated radiation therapy (IMRT) used for the management of prostate cancer (PCa).

Objectives: This study aimed at identifying the nature and severity of post-IMRT urinary symptoms in patients with PCa.

Methods: This prospective study was performed with consecutive patients, who had clinically localized PCa (cT1c-cT2c) and had undergone IMRT treatment from 2016 to 2019. At 1, 6, and 12 months of follow-up, medical history, physical information, prostatespecific antigen values, International Prostate Symptom Score (IPSS), medication use, Radiation Therapy Oncology Group (RTOG), acute and late toxicity, and Q max were collected.

Results: A total of 127 patients with a mean age of 71.04 \pm 7.1 years received IMRT and underwent 12 months of follow-up. The mean IPSSs at baseline versus those at 1, 6, and 12 months after IMRT was $14.5 \pm 6.8$ versus $13.3 \pm 6.1,12.3 \pm 5.3$, and $10.4 \pm 4.2$, respectively (P $<0.000$ ). The mean prostate volume was $38.2 \pm 12.1 \mathrm{cc}$. At the last follow-up, 31 patients (24.4\%) took genitourinary(GU) medications. Conclusions: This study showed that the majority of GU side effects caused by primary IMRT for PCa treatment are transient. Treatment triggered an acute increase in obstructive urinary symptoms, which peaked during the first month after IMRT. In most patients, in the course of 6 months, symptoms returned to baseline.
\end{abstract}

Keywords: Intensity-Modulated Radiation Therapy, Prostate Cancer, IPSS, Genitourinary Toxicities, Quality of Life

\section{Background}

For patients suffering from prostate cancer (PCa), several treatment modalities are available (1-3). An important aspect of counseling patients is to understand the impact of each management option on their quality of life (QoL) $(4,5)$. Some of these treatments, such as androgen deprivation therapy (ADT) have positive effects on QoL that improve the QoL of patients with moderate to severe voiding dysfunction via decreasing prostate volume and lower urinary tract symptoms (LUTS) $(3,6)$. Following both radiation therapy (RT) and surgery, LUTS and sexual dysfunction are common (7).

In the field of PCa treatment, when radiotherapy is compared with other treatment modalities, it is of great importance to assess the side effects caused by radiation (8). In comparison with rectal symptoms, postradiotherapy urinary symptoms affect more patients, par- ticularly with dose escalation, despite the use of more conformal management techniques, thereby drawing much more attention to themselves (9). Risk factors leading to genitourinary (GU) toxicities are not fully understood (10). Currently, there is limited evidence regarding the urinary toxicity of intensity-modulated radiation therapy (IMRT) (11). Urinary symptoms can be influenced by numerous factors, such as dosimetric characteristics and patients' comorbidities, including diabetes mellitus, baseline urinary symptoms, and hypertension (9). On the other hand, urinary symptoms caused by treatment show notable levels of reversibility linked to the contraction of both malignant and benign hypertrophic tissues, which are clinically relevant $(12,13)$.

In localized PCa subjects treated with brachytherapy, the role of pre-treatment International Prostate Symptom Score (IPSS) in estimating post-treatment urinary morbidities has been widely evaluated (14). Some studies demon- 
strated that patients, who had a combination of high IPSS before treatment and poor urinary functions, are not the ideal candidates for brachytherapy (15). IPSS was initially utilized as a patient-reported evaluation of benign prostatic hyperplasia symptoms. It benefits from good internal validity. It comprises 7 symptoms, including nocturia, frequency, weak urinary stream, intermittency, incomplete emptying, hesitancy and urgency, and also one QoL due to urinary symptoms (uQoL) question. Each answer is assigned to points from 0 to 5 and the total score can, consequently, range from 0 to 35 . The severity of symptoms is, then, divided into 3 categories according to the total score; mild (symptom score less than or equal to 7) moderate (symptom score range 8 - 19), and severe (symptom score range 20 - 35) $(16,17)$.

\section{Objectives}

Yet, the pre-treatment IPSS importance and the IPSS time course in the IMRT for PCa, have not been clearly illustrated. The aims of this research were the characterization of the nature and severity of the post-IMRT urinary functions for urinary symptoms of patients with PCa.

\section{Methods}

\subsection{Patients}

A total of 127 consecutive cases with clinically-localized PCa (cT1c-cT2c), who underwent IMRT from 2016 to 2019 were enrolled in this prospective study. All subjects received neoadjuvant hormonal therapy.

\subsection{Inclusion and Exclusion Criteria}

Patients who previously underwent prostatectomy or brachytherapy, intra-prostatic calcification of more than 1 $\mathrm{cm}$, or concomitant anal stricture were excluded from the study.

Eligible patients had newly diagnosed, biopsyproven with minimum 12-month potential follow-up, untreated, and non-metastatic PCa. No patient underwent transurethral resection of the prostate before IMRT.

\subsection{Study Endpoint}

Study endpoints included IPSS, urinary toxicities in their early and late forms, prostate-specific antigen (PSA) response, and measures based on sexual function questionnaires.

\subsection{Follow-up and IPSS Assessment}

IPSSs at baseline, and at 1, 6, and 12 months after IMRT were evaluated. In each follow-up session, medical history and physical examination, PSA values, IPSS, Q max, acute, late toxicity scores according to Radiation Therapy Oncology Group (RTOG), and medication use were accumulated. Late GU toxicities were defined as toxicities recorded 90 days after the last radiotherapy session. The use of alphaantagonists was documented at each visit.

An increase in IPSS $\geq 12$ points was defined as the "obvious deterioration" of LUTS (ODL) (18). This definition is based on the rationale and an increase in IPSS $\geq 12$ points during 3 months brings the patients with mild or intermediate levels to the verge of higher IPSS levels.

\subsection{Radiation Therapy}

Varian Clinac 600C (Varian Inc., California, USA) was utilized to deliver radiotherapy by using an X-ray beam of $6 \mathrm{MV}$. Using a 9-field technique, patients with localized PCa, who were definitive RT candidates, received hypofractionated (70.2 Gy, 2.7 Gy/fraction) IMRT (19). In these patients, the entire prostate gland and seminal vesicles were defined as clinical target volume (CTV). The extension of CTV of $0.6 \mathrm{~cm}$ posteriorly and $1 \mathrm{~cm}$ in all other directions was considered planning target volume (20). Three gold fiducial markers were implanted for daily interfraction guidance, at least 1 week before simulation. Coregistration with pelvic MRI was done for all patients. The daily setup was verified by an electronic portal imaging device (EPID). Treatment planning was performed, using the Eclipse treatment planning system (Varian Medical System).

All patients discharged urine and, then, drank $250 \mathrm{cc}$ water 1 hour before the CT scan and each treatment (21). Special regimens were given to patients for bowel preparation (22).

Treatment was designed according to a treatment planning; supine positioned CT scan with $3 \mathrm{~mm}$ slice thickness.

\subsection{Statistics}

The effects of subject characteristics and management parameters on the IPSS difference between baseline and 12 months after intervention were analyzed by the MannWhitney $U$ test and the multiple linear regression models. Sample medians and ranges were used to describe continuous variables. The Friedman test was used to detect the changes of IPSS from baseline to 12 months after IMRT. The level of statistical significance was set at $\mathrm{P}<0.05$. 


\subsection{Ethics}

The Ethics Committee of Shahid Beheshti University of Medical Sciences approved the protocol of this study. All the patients were informed of the scientific nature of the study and written informed consent was obtained from all subjects.

\section{Results}

Baseline characteristics of patient and disease are illustrated in Table 1.

The IPSS average was the highest at baseline (14.5 \pm 6.8$)$. The mean QoL score and IPSS for the entire cohort trend in follow-up visits showed a statistical difference compared with baseline. The mean IPSS score of patients in pretreatment, 3, 6, and 12 months after IMRT were shown in Table 2.

During IMRT, 31 patients (24.4\%) started GU medications (alpha-blockers). Of 18 patients (14.1\%), who had shown acute Grade 2 urinary toxicity, most of the manifested symptoms were comprised of urinary frequency and dysuria.

\begin{tabular}{|c|c|c|}
\hline Parameter & Mean \pm SD & Percentage \\
\hline $\operatorname{Age}(y / 0)$ & $71.04 \pm 7.1$ & \\
\hline Min & & $55 \mathrm{y} / 0$ \\
\hline Max & & $87 \mathrm{y} / 0$ \\
\hline Median prostate volume $(\mathrm{mL})$ & $38.2 \pm 12.1$ & \\
\hline Min & & 20 \\
\hline Max & & 60 \\
\hline Bladder volume (mL) & $280.07 \pm 54.2$ & \\
\hline Min & & 160 \\
\hline Max & & 430 \\
\hline BMI $\left(\mathrm{kg} / \mathrm{cm}^{2}\right)$ & $27.1 \pm 4.7$ & \\
\hline Min & & 19.9 \\
\hline $\operatorname{Max}$ & & 37.4 \\
\hline Gleason sum & $7.1 \pm 0.9$ & \\
\hline \multicolumn{3}{|l|}{ Clinical T stage } \\
\hline $\mathrm{T} 1-\mathrm{T} 2$ & 84 & 66.1 \\
\hline $\mathrm{T} 3-\mathrm{T} 4$ & 43 & 33.9 \\
\hline \multicolumn{3}{|l|}{ Risk groups (D'Amico's) } \\
\hline Low & 68 & 53.5 \\
\hline Intermediate & 35 & 27.5 \\
\hline High & 24 & 19 \\
\hline
\end{tabular}

\section{Discussion}

Urinary function, sexual function, and PSA response to treatment play significant roles in the management of post-RT prostate cancer patients (18). In PCa patients, urinary symptoms following radiotherapy are drawing much more attention in comparison with rectal symptoms as they affect more patients (23).

Consistent with previous studies, baseline symptoms had the strongest predictive value for frequency and incontinence (24). In evaluating the radiotherapy effects, some conditions are almost exclusive to urinary symptoms. The LUTS are sometimes unclear from symptoms related to management, and their prevalence is rising with age (25).

In IMRT, for better bladder tolerance, strictly conformal-dynamic techniques of radiotherapy with precise fiducial-based positioning, which are limited exclusively to the prostate gland, may be beneficial (26).

The results of this study proposed a negligible impact on patients' QoL due to high-dose radiation therapy for PCa. Similar to other studies, decreasing symptoms of endpoints is potentially related to the amelioration of the symptoms after radiotherapy treatment $(9,14,27)$.

A study of 368 patients concerning urinary outcomes from Chicago Hospital, who were mainly treated with IMRT with a median dose of $75.6 \mathrm{~Gy}$, showed median IPSS alterations from first to final follow-up worsened by 1 point and improved by 2 and 11 points in patients with mild, moderate, and severe baseline IPSS, respectively (14). A report from Nagoya of 216 subjects treated with IMRT to 74-78 Gy presents comparable outcomes at 2 years with mean IPSS alterations of $+1.6,2.9$, and 10.3 from baseline in patients with mild, moderate, and severe baseline IPSS, respectively (10).

A proper assessment of dose-volume GU toxicity depends on the accurate bladder filling between CT simulation and irradiation (10). In the present investigation, all cases discharged urine approximately 1 hour before IMRT and CT simulation; they also defecated every morning when possible. These measures were considered to minimize the effects of variations in the prostate shape and anatomical location.

Some studies have shown acute deterioration of QoL and urinary symptoms based on IPSS (28) that improved within 1 to 2 years after the treatment $(29,30)$. A study conducted in the Memorial Sloan Kettering Cancer Centre (MSKCC) utilizing IPSS and CTCAE reported urinary outcomes in 268 subjects and showed a decline in doseescalated IMRT/IGRT (29). In line with the aforementioned study, this cohort study showed improvement in posttreatment median IPSS over time. However, there are differences in sample size, the total dose of radiotherapy, as 


\begin{tabular}{|c|c|c|c|c|c|}
\hline Variable & Baseline & 1 Month & 6 Months & 12 Months & P Value \\
\hline $\operatorname{PSA}(\mathrm{ng} / \mathrm{dL})$ & $10 \pm 3.2$ & $3 \pm 1.4$ & $0.9 \pm 0.8$ & $0.2 \pm 0.5$ & 0.00 \\
\hline IPSS & $13.3 \pm 6.1$ & $14.5 \pm 6.8$ & $12.3 \pm 5.3$ & $10.4 \pm 4.2$ & 0.00 \\
\hline IPSS QOL & $3.2 \pm 1.6$ & $2.8 \pm 1.2$ & $2.5 \pm 1$ & $1.9 \pm 0.7$ & 0.01 \\
\hline IPSS $\mathrm{i}$ & $7.2 \pm 3.4$ & $7.4 \pm 3.6$ & $6.7 \pm 3.2$ & $5.3 \pm 2.3$ & 0.00 \\
\hline IPSS o & $6.1 \pm 3$ & $7.1 \pm 3.5$ & $5.6 \pm 2.3$ & $5.1 \pm 2$ & 0.005 \\
\hline IIEF & $12.7 \pm 3.9$ & $12.2 \pm 3.2$ & $13.4 \pm 3.6$ & $14.5 \pm 3.2$ & 0.00 \\
\hline Q Max & $15 \pm 6.5$ & $15.6 \pm 5.5$ & $18.2 \pm 5.2$ & $20 \pm 4.8$ & 0.00 \\
\hline
\end{tabular}

Abbreviations: IPSS i, Irritative IPSS; IPSS o, Obstructive IPSS; IIEF, International Index of Erectile Function.

well as the difference in the dosage of each treatment session, and bladder filling radiotherapy between the present and the MSKCC study.

Over time, the urinary symptom may improve due to the following mechanisms: (1) Using GU medications, (2) Disease burden reduction, (3) Neo-adjuvant RT and/or ADT causing prostate cytoreduction, and (4) patient bias $(14,31)$.

The most important limitation of this research is the small sample size of the study. Short-term follow-up and the lack of prostate size measurement during the treatment period are other limitations.

\subsection{Conclusions}

The findings of this study demonstrate that this procedure is safe concerning LUT function. Also, it shows that the majority of GU side effects after primary IMRT for prostate cancer are transient. A rapid increase was observed regarding urinary symptoms peaked at 1 month after IMRT. In most patients in the course of 6 months, symptoms returned to baseline.

The risk of acute urinary retention that was resulted in catheterization was low, although delayed deterioration in obstructive symptoms was common and mostly transient. Factors thought to have an impact on the IPSS changes include GU medications, baseline IPSS, Age, and prostate tumor burden.

\section{Footnotes}

Authors' Contribution: Study concept and design: Farzad Allameh , Amir Reza Abedi; Acquisition of data: Morteza Fallah Karkan, and Amirhosein Rahavian; analysis and interpretation of data: Saeed Montazeri and Samira Azghandi; drafting of the manuscript: Bahram Mofid; critical revision of the manuscript for important intellectual content: Abbas Basiri; statistical analysis: Morteza Fallah Karkan and Amir Reza Abedi; administrative, technical, and material support: Amir Reza Abedi; study supervision: Farzad Allameh.

Conflict of Interests: The authors declare no conflict of interest regarding the publication of this paper.

Ethical Approval: The Ethical Committee of Shahid Beheshti University of Medical Sciences approved the protocol of this study.

Funding/Support: This research did not receive any specific grant from funding agencies in the public, commercial, or not-for-profit sectors.

Patient Consent: All the patients were informed of the scientific nature of the study, and written informed consent were obtained from all subjects.

\section{References}

1. Ghiasy S, Abedi AR, Moradi A, Hosseini SY, Karkan MF, Sadri G, et al. Is active surveillance an appropriate approach to manage prostate cancer patients with Gleason Score 3+ 3 who met the criteria for active surveillance? Turkish journal of urology. 2019;45(4):261.

2. Salehi B, Fokou PVT, Yamthe LRT, Tali BT, Adetunji CO, Rahavian A, et al. Phytochemicals in Prostate Cancer: From Bioactive Molecules to Upcoming Therapeutic Agents. Nutrients. 2019;11(7):1483.

3. Ahmadi H, Daneshmand S. Androgen deprivation therapy for prostate cancer: long-term safety and patient outcomes. Patient related outcome measures. 2014;5:63.

4. Chin S, Hayden AJ, Gebski V, Cross S, Turner SL. Long term patient reported urinary function following external beam radiotherapy for prostate cancer. Clinical Oncology. 2017;29(7):421-8.

5. Pourmand G, Salem S, Mehrsai A, Lotfi M, Amirzargar MA, Mazdak H, et al. The risk factors of prostate cancer: a multicentric case-control study in Iran. Asian Pacific Journal of Cancer Prevention. 2007;8(3):4228.

6. Axcrona K, Aaltomaa S, Da Silva CM, Özen H, Damber J, Tankó LB, et al. Androgen deprivation therapy for volume reduction, lower urinary tract symptom relief and quality of life improvement in patients with prostate cancer: degarelix vs goserelin plus bicalutamide. BJU international. 2012;110(11):1721-8.

7. Rana Z, Hong RL, Abugideiri M, McRae D, Cernica G, Mordkin R, et al. Sexual, irritative, and voiding outcomes, following stereotactic body radiation therapy for prostate cancer. Radiation Oncology. 2015;10(1):182. 
8. Goldner G, Wachter-Gerstner N, Wachter S, Dieckmann K, Janda M, Pötter R. Acute side effects during 3-D-planned conformal radiotherapy of prostate cancer. Strahlentherapie und Onkologie. 2003;179(5):320-7.

9. Yahya N, Ebert MA, Bulsara M, Haworth A, Kennedy A, Joseph DJ, et al. Dosimetry, clinical factors and medication intake influencing urinary symptoms after prostate radiotherapy: An analysis of data from the RADAR prostate radiotherapy trial. Radiotherapy and Oncology. 2015;116(1):112-8.

10. Tomita N, Oze I, Shimizu H, Yoshida M, Kimura K, Takehana K, et al. International prostate symptom score (IPSS) change and changing factor in intensity-modulated radiotherapy combined with androgen deprivation therapy for prostate cancer. Nagoya journal of medical science. 2015;77(4):637.

11. Viswanathan AN, Yorke ED, Marks LB, Eifel PJ, Shipley WU. Radiation dose-volume effects of the urinary bladder. International Journal of Radiation Oncology* Biology* Physics. 2010;76(3):S116-22.

12. Yahya N, Ebert MA, Bulsara M, House MJ, Kennedy A, Joseph DJ, et al. Urinary symptoms following external beam radiotherapy of the prostate: Dose-symptom correlates with multiple-event and eventcount models. Radiotherapy and Oncology. 2015;117(2):277-82.

13. Schmid MP, Pötter R, Bombosch V, Sljivic S, Kirisits C, Dörr W, et al. Late gastrointestinal and urogenital side-effects after radiotherapy-Incidence and prevalence. Subgroup-analysis within the prospective Austrian-German phase II multicenter trial for localized prostate cancer. Radiotherapy and Oncology. 2012;104(1):114-8.

14. Malik R, Jani AB, Liauw SL. External beam radiotherapy for prostate cancer: urinary outcomes for men with high International Prostate Symptom Scores (IPSS). International Journal of Radiation Oncology* Biology* Physics. 2011;80(4):1080-6.

15. Nag S, Beyer D, Friedland J, Grimm P, Nath R. American Brachytherapy Society (ABS) recommendations for transperineal permanent brachytherapy of prostate cancer. International Journal of Radiation Oncology* Biology* Physics.1999;44(4):789-99.

16. Barry MJ, Fowler FJ, O'Leary MP, Bruskewitz RC, Holtgrewe HL, Mebust WK, et al. The American Urological Association symptom index for benign prostatic hyperplasia. The Journal of urology. 1992;148(5 Part 1):1549-57.

17. Gutman S, Merrick GS, Butler WM, Wallner KE, Allen Z, Galbreath RW, et al. Severity categories of the International Prostate Symptom Score before, and urinary morbidity after, permanent prostate brachytherapy. BJU international. 2006;97(1):62-8.

18. Fukazawa A, Koiwai K, Ozawa T, Matsushita H, Kadoya M. Predictive Factors for Deterioration of Lower Urinary Tract Symptoms After Iodine-125 Brachytherapy in Prostate Cancer Patients. The Shinshu Medical Journal. 2018;66(2):131-7.

19. Pollack A, Hanlon AL, Horwitz EM, Feigenberg SJ, Konski AA, Movsas $\mathrm{B}$, et al. Dosimetry and preliminary acute toxicity in the first 100 men treated for prostate cancer on a randomized hypofractionation dose escalation trial. International Journal of Radiation Oncology* Biology*
Physics. 2006;64(2):518-26.

20. Chung HT, Xia P, Chan LW, Park-Somers E, Roach III M. Does image-guided radiotherapy improve toxicity profile in whole pelvictreated high-risk prostate cancer? Comparison between IG-IMRT and IMRT. International Journal of Radiation Oncology* Biology* Physics. 2009;73(1):53-60.

21. Holden L, Stanford J, D'Alimonte L, Kiss A, Loblaw A. Timing variability of bladder volumes in men receiving radiotherapy to the prostate. Journal of medical imaging and radiation sciences. 2014;45(1):24-30.

22. Jones CU, Hunt D, McGowan DG, Amin MB, Chetner MP, Bruner DW, et al. Radiotherapy and short-term androgen deprivation for localized prostate cancer. New England Journal of Medicine. 2011;365(2):107-18.

23. Zelefsky MJ, Levin EJ, Hunt M, Yamada Y, Shippy AM, Jackson A, et al. Incidence of late rectal and urinary toxicities after three-dimensional conformal radiotherapy and intensity-modulated radiotherapy for localized prostate cancer. International Journal of Radiation Oncology* Biology* Physics. 2008;70(4):1124-9.

24. Li X, Fang D, Cooperberg MR, Whitson JM, Lue TF, Zhou L, et al. Long-term follow-up of International Prostate Symptom Score (IPSS) in men following prostate brachytherapy. World journal of urology. 2014;32(4):1061-6.

25. Malmsten UG, Molander U, Peeker R, Irwin DE, Milsom I. Urinary incontinence, overactive bladder, and other lower urinary tract symptoms: a longitudinal population-based survey in men aged 45-103 years. European urology. 2010;58(1):149-56.

26. Majewski W, Tabor K, Prokop E, Kulik R. Quality of life in patients with prostate cancer treated with radical image-guided radiotherapy. Contemporary Oncology. 2014;18(4):285.

27. Christie D, Denham J, Steigler A, Lamb D, Turner S, Mameghan $\mathrm{H}$, et al. Delayed rectal and urinary symptomatology in patients treated for prostate cancer by radiotherapy with or without short term neo-adjuvant androgen deprivation. Radiotherapy and oncology. 2005;77(2):117-25.

28. Burden HP, Evans S, Jones R, Graham J, Persad R. Acute urinary morbidity during $3 \mathrm{D}$ conformal radiotherapy for localised prostate cancer. British Journal of Medical and Surgical Urology. 2009;2(2):62-6.

29. Ghadjar P, Jackson A, Spratt DE, Oh JH, af Rosenschöld PM, Kollmeier $\mathrm{M}$, et al. Patterns and predictors of amelioration of genitourinary toxicity after high-dose intensity-modulated radiation therapy for localized prostate cancer: implications for defining postradiotherapy urinary toxicity. European urology. 2013;64(6):931-8.

30. Lee WR, Hall MC, McQuellon RP, Case LD, McCullough DL. A prospective quality-of-life study in men with clinically localized prostate carcinoma treated with radical prostatectomy, external beam radiotherapy, or interstitial brachytherapy. International Journal of Radiation Oncology* Biology* Physics. 2001;51(3):614-23.

31. Mearini L, Nunzi E, Giovannozzi S, Lepri L, Lolli C, Giannantoni A. Urodynamic evaluation after high-intensity focused ultrasound for patients with prostate cancer. Prostate cancer. 2014;2014. 